\title{
Revisión sistemática de la investigación sobre el uso del mindfulness en la educación física
}

\section{Systematic review of research on the use of mindfulness in physical education}

\section{Revisão sistemática de pesquisas sobre o uso da atenção plena na educação física}

\author{
López, I. ${ }^{1}$, Gené-Morales, J. ${ }^{2}$ \\ ${ }^{1}$ Florida Universitària; Universidad de Valladolid ${ }^{2}$
}

\begin{abstract}
RESUMEN
Objetivo: Esta revisión sistemática tuvo el objetivo de revisar los artículos publicados en los últimos seis años sobre el uso de mindfulness en la educación física. Método: Se analizaron los artículos publicados desde 2014 hasta 2020 mediante las bases de datos ERIC, Taylor y Francis, Web of Science y SCOPUS. Resultados: Se han incluido 15 artículos. De estos artículos la mayoría fueron publicados en Estados Unidos, sin ninguna representación en el España. Los resultados confirman los beneficios del mindfulness en la mejora del desarrollo de la educación emocional, la atención plena, habilidades de conciencia, la autoeficacia, la autorregulación, la disminución del estrés, del rendimiento deportivo y la actividad física. Conclusiones: A pesar de los beneficios corroborados del mindfulness en el ámbito deportivo, en España, en comparación con otros países, las investigaciones sobre mindfulness y educación física escolar son prácticamente inexistentes.
\end{abstract}

Palabras clave: mindfulness, psicología, deporte, educación física

\begin{abstract}
Objective: This systematic review aimed to review the articles published in the last six years on the use of mindfulness in physical education. Method: Articles published from 2014 to 2020 were analyzed using the ERIC, Taylor and Francis, Web of Science and SCOPUS databases. Results: 15 articles have been included. Most of these articles were published in the United States, without any representation in Spain. The results confirm the benefits of mindfulness in improving the development of emotional education, mindfulness, awareness skills, self-efficacy, self-regulation, reduction of stress, sports performance and physical activity. Conclusions: Despite the corroborated benefits of mindfulness in sports, in Spain, compared to other countries, research on mindfulness and school physical education is practically non-existent.
\end{abstract}

Keywords: mindfulness, psychology, sport, physical education

\section{RESUMO}

Objetivo: Esta revisão sistemática teve como objetivo revisar os artigos publicados nos últimos seis anos sobre o uso da mindfulness na educação física. Método: Os artigos publicados de 2014 a 2020 foram analisados nas bases de dados ERIC, Taylor e Francis, Web of Science e SCOPUS. Resultados: 15 artigos foram incluídos. A maioria desses 


\section{López Secanell, I.; Gené-Morales, J.}

artigos foi publicada nos Estados Unidos, sem nenhuma representação na Espanha. Os resultados confirmam os benefícios da atenção plena na melhoria do desenvolvimento da educação emocional, atenção plena, habilidades de consciência, autoeficácia, autorregulação, redução do estresse, desempenho esportivo e atividade física. Conclusões: Apesar dos benefícios comprovados da mindfulness no esporte, na Espanha, em comparação com outros países, as pesquisas sobre mindfulness e educação física escolar são praticamente inexistentes.

Palavras chave: atenção plena, psicologia, desporto, educação física

\section{INTRODUCCIÓN}

En los últimos años hemos vivido un claro aumento de la implementación y las investigaciones en mindfulness dentro de la psicología del deporte (Palmi y Solé, 2016). La definición más utilizada del mindfulness es la de Kabat-Zinn (2003): "el mindfulness es el estado particular de conciencia que surge al poner la atención, de forma intencional y sin juzgar, en el momento presente, viviendo la experiencia momento a momento" (p.145).

La principal justificación de este incremento se ha basado en los beneficios de utilizar el mindfulness para mejorar la salud mental (física, social, emocional y cognitiva) de los estudiantes (Ergas y Hadar, 2019). Este incremento de la investigación en torno a esta técnica ha permitido evidenciar algunos de sus beneficios a nivel psicológico, emocional y cognitivo. Respecto a los beneficios psicológicos encontramos su influencia en la reducción de los niveles de estrés (Anand y Sharma, 2014; Kuyken et al., 2013; Metz et al., 2013), de ansiedad (Potek, 2012; Napoli, Krech y Holley, 2005; So y Orme-Johnson, 2001), de depresión (Schonert-Reichl et al., 2015; Britton et al., 2014; Kuyken et al., 2013), de efecto negativo (Britton et al., 2014; Schonert-Reichl y Lawlor, 2010; Broderick y Metz, 2009) y de cansancio, dolor y quejas psicosomáticas (Metz et al., 2013; Broderick y Metz, 2009). A nivel emocional se constata una mayor conciencia y claridad emocional (Schonert-Reichl et al., 2015; Broderick y Metz, 2009) y regulación de emociones negativas (Schonert-Reichl et al., 2015; Broderick y Metz, 2009; Rosaen y Benn, 2006). A nivel cognitivo los beneficios que se observan se centran en el aumento en los niveles de atención (Tang, Yang, Leve y Harold, 2012; Schonert-Reichl y Lawlor, 2010; Saltzman y Goldin, 2008; Napoli et al., 2005), aumento en la autorregulación y función ejecutiva (Tang et al., 2012; Biegel y Brown, 2010; Flook et al., 2010; Desmond, Hanich y Millersville,
2010), aumento de velocidad de procesamiento de información, inteligencia práctica, independencia de campo y creatividad (So y Orme-Johnson, 2001) y en el incremento de los niveles de creatividad verbal (Franco, 2009).

Los beneficios que ha presentado el mindfulness en el ámbito de la psicología han hecho que en los últimos años esta técnica sea transferida a otros ámbitos, entre los que encontramos el deporte y la educación física (EF) (Solé et al., 2014)

\section{Mindfulness, deporte y EF}

El interés de la psicología por el mindfulness se ha justificado por los numerosos beneficios mencionados anteriormente, lo cual ha conllevado a poner en práctica esta técnica en el contexto deportivo mediante el diseño de intervenciones y también diversos programas. Uno de ellos es el Mindful Sport Performance Enhacement (MSPE) de Kauffman, Glass y Arnkoff (2009), centrado en la mejora del rendimiento deportivo a través de la conciencia plena. Éste se focaliza en enseñar la aplicación del mindfulness en el deporte mediante la práctica repetida y la meditación adaptada a cada juego. El programa fue utilizado en otras investigaciones como la de Minkler, Glass y Hut, (2020), los cuales lo implementaron durante 6 semanas en 30 atletas femeninas de tercera división de lacrosse de la National Collegiate Athletic Association. La investigación concluyó mejoras significativas en la ansiedad, la consciencia, dificultades en la regulación de las emocionales y el rendimiento deportivo y la satisfacción. Además, concluye la importancia de que los profesionales de la psicología deportiva formen a los entrenadores para integrar el mindfulness en sus contextos deportivos.

Otro programa es el MAC (Mindfulness AcceptanceCommitment) de Gardner y Moore (2004) que tiene un 


\section{Revisión sistemática sobre mindfulness en la EF}

enfoque basado en la atención plena y se organiza en cinco componentes: identificación de valores y compromiso, aceptación, integración, psicoeducación y práctica. Los principales resultados de este programa son una reducción en la ansiedad deportiva (Lutkenhouse, 2007; Schwanhausser, 2009), aumento en el rendimiento (Lutkenhouse, Gardner y Moore, 2007), un aumento significativo en las calificaciones de rendimiento atlético la atención centrada en la tarea y la intensidad de la práctica (Wolanin, 2005), aumentos significativos en la capacidad para describir los pensamientos y emociones de los jugadores, aceptar experiencias del momento presente al reaccionar ante ellos y comprometerse con comportamientos directamente relacionados con el logro de sus metas atléticas (Hasker, 2010).

Otro de los programas principales es "Meditación Fluir" de Franco (2009), el cual se realiza con futbolistas y constata como el trabajo con mindfulness resulta eficaz en la prevención de lesiones deportivas y en la mejora de la rehabilitación.

Según Solé, Carrança, Serpa y Palmi (2014) el mindfulness debe ser una herramienta que se siga extendiendo en el ámbito deportivo, dado que en otros gremios como el psicológico queda demostrada su competencia. Las investigaciones realizadas evidencian como el uso del mindfulness en el deporte favorece una mejora del rendimiento del deportista (Palmi y Solé, 2016; Gooding y Gardner, 2009). Además, se han constatado mejoras en las expectativas de los deportistas hacia la competición (Kauffman et al., 2009; Palmi y Solé, 2016; Worthen y Luiselli, 2016), así como mejoras en su relajación (Dunn y Hartigan, 1999; Kauffman et al., 2009), atención y consciencia durante el juego (Worthen y Luiselli, 2016; Cox, Ullrich-French, y French, 2016), variables psicológicas como la ansiedad (Kauffman et al., 2009; Palmi y Solé, 2016; Nolte, Steyn, Krüger y Fletcher, 2016; Gross et al. 2016) y también una reducción del "burnout" en deportistas (Garcés, Palacios y Arce, 2012).

Según lo expuesto, el uso del mindfulness tiene numerosos beneficios en el deporte profesional. No obstante, nos encontramos con una falta de estudios que evidencien si esta técnica también se está utilizando en la EF escolar, así como sus beneficios en este ámbito. La relación entre la EF y el mindfulness es evidente y cercana (Del Águila et al., 2009), ya que a nivel curricular esta técnica se relaciona con los contenidos de relajación y respiración (Pérez y Botella, 2006; Delgado, 2009), convirtiéndose en un contenido transversal que busca el desarrollo integral de la persona (Beltrán y Abad, 2011). Un ejemplo de ello es el estudio realizado por Yook, Kang y Park (2017), quienes incluyeron el mindfulness en los juegos del kinball y el yoga en 46 alumnos de primaria durante 8 semanas. El resultado de la experiencia concluyó que el mindfulness mejora la autoestima, la resiliencia y la felicidad en los deportistas. También es un ejemplo la experiencia realizada por Mulhearn, Kulinna y Lorenz (2017) quienes exploran los beneficios del mindfulness en la EF para los alumnos de 12 años concluyendo un aumento del rendimiento en sus habilidades, una disminución de problemas de comportamiento y un aumento de la consciencia cenestésica.

Aunque la relación entre EF y mindfulness es evidente, nuestra hipótesis es que son pocos los estudios realizados a nivel español donde se utilice esta técnica en contextos educativos y concretamente en el ámbito deportivo de la EF. Por este motivo, el objetivo de este trabajo es realizar una revisión sistemática de los artículos publicados en los últimos seis años sobre el uso de mindfulness en la EF de los distintos niveles educativos (educación infantil, primaria, secundaria y formación profesional), así como sus principales conclusiones.

\section{MATERIAL Y MÉTODOS}

Este trabajo consiste en una revisión sistemática de artículos de investigación empírica y/o instrumental (Ato, López y Benavente, 2013) sobre el uso del mindfulness en clases de EF de centros educativos publicados en los últimos seis años (2014-2020) con estudiantes de educación infantil, primaria, secundaria y formación profesional. Para realizar el planteamiento del estudio nos hemos basado en el diseño de investigación realizada por Barba, Bores, Hortigüela y González (2020) donde se realiza una la revisión sistemática utilizando la pauta PRISMA y la estrategia PICO. PRISMA propone 27 elementos y un diagrama de flujo para facilitar la presentación de informes de revisión bibliográfica y meta-análisis (Urrútia y Bonfill, 2010). En el caso de este estudio se respeta su uso teniendo en cuenta los elementos clave de esta pauta como la terminología, la formulación de 


\section{López Secanell, I.; Gené-Morales, J.}

pregunta de investigación, la identificación de los estudios, la extracción de datos, la calidad de los estudios, etc. Respecto a PICO, es una estrategia que nos permite plantear preguntas teniendo en cuenta la definición del problema (P), la intervención a analizar (I), comparación de intervenciones (C) y resultados (outcomes) (O), los cuales nos ha permido estructurar el diseño de la investigación, así como plantear la hipótesis del trabajo. En nuestro estudio, las bases de datos utilizadas han sido: ERIC, Taylor y Francis, Web of Science y SCOPUS. Los descriptores utilizados fueron "mindfulness", "atención plena" y "educación física".

Junto a las bases de datos y los descriptores, se han concretado los siguientes criterios de exclusión:

- Artículos duplicados

- Artículos sobre mindfulness que no se aplican en clases de EF.

- Artículos no publicados en revistas indexadas en JCR o SJR.

- Artículos en lenguajes distintos de ingles o español.

- Artículos en que el mindfulness no se implementa en centros educativos (escuelas de infantil, primaria secundaria o/y formación profesional)

- $\quad$ Artículos en que el mindfulness se implementa en universidades.

\section{Procedimiento}

La investigación se inició con la selección de los criterios de inclusión y exclusión de los artículos, así como la selección de las bases de datos. Respecto a los criterios de inclusión, consideramos que debíamos incorporar el descriptor "atención plena" junto al de "mindfulness", mediante el operador booleano OR, ya que la revisión teórica realizada nos evidenciaba que en el estado español se consideran conceptos sinónimos. Junto a estos descriptores se seleccionó el de "Educación física", ya que queríamos focalizar la búsqueda de artículos en esta materia y en el contexto escolar. Este término clave se relacionó con los dos anteriores mediante el operador booleano AND para recoger el mayor número de resultados posibles $\mathrm{y}$ pasar los criterios de exclusión que se han presentado anteriormente. Estos criterios de exclusión se diseñaron teniendo en mente los criterios de inclusión del estudio.
Una vez concretados los criterios, se seleccionaron las bases de datos que se utilizarían para realizar la revisión (ERIC, Taylor y Francis, Web of Science y SCOPUS). Justificamos la elección de dichas bases principalmente por su prestigio internacional.

Con la concreción de los criterios y las bases de datos se procedió a iniciar la investigación, encontrando un total de 224 publicaciones que correspondían a los descriptores mencionados (ver figura 1). Dicha búsqueda bibliográfica fue realizada por la investigadora principal, la cual fue la responsable de leer los resúmenes de los textos para proceder a la aplicación de los primeros criterios de exclusión e inclusión y realizar el análisis de contenido. Después de la aplicación de los primeros criterios de exclusión se seleccionaron 40 artículos, ya que la mayoría de ellos se aplicaban en contextos de rendimiento y no en contextos escolares. Finalmente, con la aplicación de los segundos criterios de exclusión se descartaron aquellos artículos que eran revisiones y se seleccionaron los 15 artículos que forman parte de esta investigación. Para analizar los criterios de calidad de los artículos seleccionados se procedió a utilizar la Tabla 1 por parte de la investigadora principal y dos colaboradores externos expertos en psicología y deporte para, por consenso entre profesionales del ámbito, evaluar la calidad de los artículos a incluir. La tabla contenía los siguientes ítems: grado de inclusión en JCR o SJR, el detalle del proceso metodológico utilizado, el número de participantes, la duración y el nivel de calidad JCR. Cada investigador cumplimentó la tabla individualmente y después la compartió con los compañeros para diseñar la tabla final. Finalmente, por consenso entre los autores, los cuáles tienen cierta experiencia en la investigación el ámbito, se creó la tabla final que incluía todos los artículos.

Una vez realizada la Tabla 1 , se procedió a diseñar la Tabla 2, con los artículos seleccionados para la investigación. Dicha tabla incluye las siguientes categorías: Autor y año, país, idioma, nivel educativo y número de participantes, duración, tipo de investigación (cualitativo, cuantitativo, etc.), objetivos, medidas, resultados, programa de mindfulness utilizado y tamaño del efecto 


\section{Revisión sistemática sobre mindfulness en la EF}

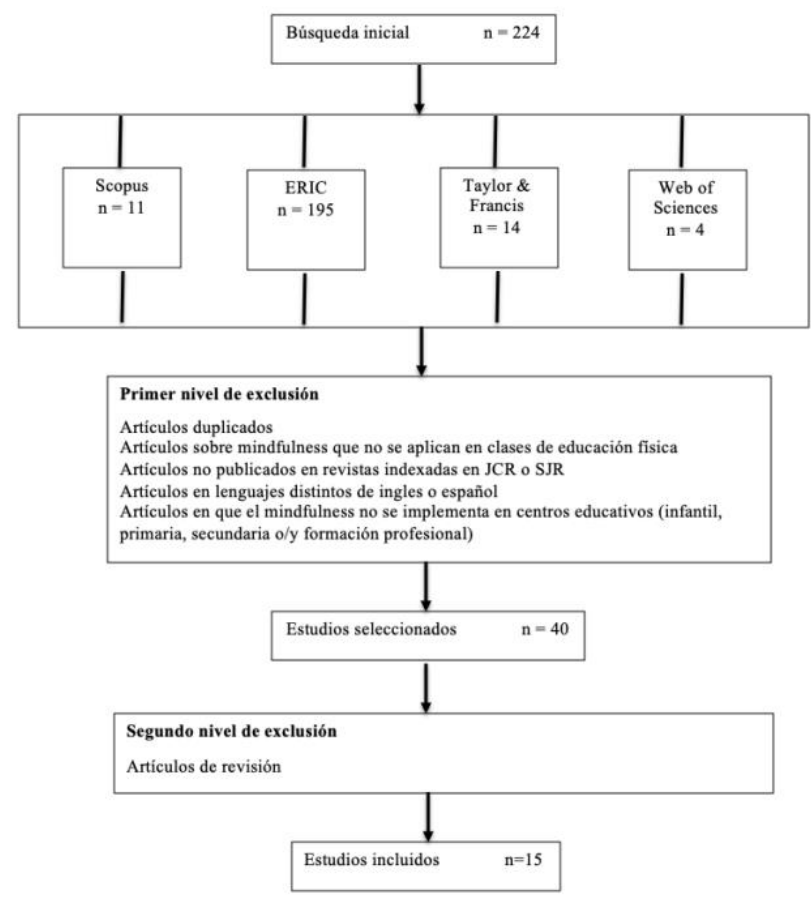

Figura 1. Diagrama de flujo para la selección

Tabla 1. Calidad de los artículos seleccionados

\begin{tabular}{|c|c|c|c|c|c|}
\hline Trabajo & $\begin{array}{c}J C R \\
/ \\
S J R \\
\end{array}$ & Metodología & Duración & $\begin{array}{c}\text { Muestra } \\
\text { total }\end{array}$ & $\begin{array}{c}\text { Nivel de } \\
\text { calidad }\end{array}$ \\
\hline $\begin{array}{l}\text { Kiens y } \\
\text { Larsen }\end{array}$ & 1 & 2 & 2 & 1 & SJR \\
\hline $\begin{array}{l}\text { (2020) } \\
\text { Minkler } \\
\text { et al. } \\
(2020)\end{array}$ & 1 & 1 & 0 & 1 & SJR \\
\hline $\begin{array}{l}\text { Firth- } \\
\text { Clark et } \\
\text { al. (2019) }\end{array}$ & 0 & 2 & 1 & 2 & $M Q S$ \\
\hline $\begin{array}{l}\text { Khng } \\
\text { (2018) }\end{array}$ & 0 & 0 & - & - & $H Q S$ \\
\hline $\begin{array}{c}\text { Petterson } \\
\text { y Olson } \\
\text { (2017) }\end{array}$ & 1,2 & 0 & - & - & $S J R / J C R$ \\
\hline $\begin{array}{c}\text { Yook et } \\
\text { al. (2017) }\end{array}$ & 1,2 & 2 & 0 & 1 & $S J R / J C R$ \\
\hline $\begin{array}{c}\text { Worthen } \\
\text { y Luiselli } \\
\text { (2016) }\end{array}$ & 1,2 & 2 & 1 & 1 & $\begin{array}{l}\text { SJR/ } \\
J C R\end{array}$ \\
\hline $\begin{array}{l}\text { Nolte et } \\
\text { al. (2016) }\end{array}$ & 1 & 2 & 0 & 2 & $S J R$ \\
\hline $\begin{array}{l}\text { Gross et } \\
\text { al. (2016) }\end{array}$ & 1,2 & 1 & - & 1 & $S J R / J C R$ \\
\hline $\begin{array}{l}\text { Ullrich- } \\
\text { French et } \\
\text { al. (2017) }\end{array}$ & 1,2 & 1 & - & 2 & $S J R / J C R$ \\
\hline $\begin{array}{c}\text { Hagins y } \\
\text { Rundle } \\
\text { (2016) }\end{array}$ & 1 & 2 & 2 & 2 & SJR \\
\hline $\begin{array}{l}\text { Meixner } \\
\text { et al. } \\
(2019)\end{array}$ & 1,2 & 1 & 2 & 2 & $\begin{array}{l}S J R / \\
J C R\end{array}$ \\
\hline
\end{tabular}

Inclusión de JCR / SJR (ifue el estudio publicado en un diario indexado en JCR o SJR?): O: no indexado, 1: indexado en SJR y 2: indexado en JCR; Metodología (¿el informe informó en detalle el proceso metodológico utilizado?): 0: no informado, 1 : informado pero impreciso (no completamente), y 2: descripción exhaustiva informada; Muestra (número de participantes): 0: menos de 10 participantes, 1 : de 10 a 50 participantes, y 2: más de 50 participantes; Duración: 0: menor que ocho lecciones, 1 : de nueve a 14 lecciones, y 2: más de 15 lecciones. Nivel de calidad JCR: informe de citas de revistas; SJR: Scimago rango de diario; HQS: estudio de alta calidad (7-10), MQS: estudio de calidad moderada (5-7).

\section{RESULTADOS}

A continuación, se discuten los 15 artículos seleccionados de los últimos seis años en torno a los ítems utilizados en la Tabla 2, a excepción del año, ya que los textos seleccionados forman parte de un mismo periodo de tiempo. Algunos de los ítems utilizados se han agrupado en un mismo apartado a causa de la relación entre ellos.

\section{País e idioma}

Los resultados muestran una clara predominancia de los Estados Unidos, representando a 7 de los 15 artículos seleccionados. Este resultado va en consonancia con el idioma inglés, el cual representa el $100 \%$ de los resultados obtenidos. En 2007 los estadounidenses invirtieron 4 millones de dólares en mindfulness para mejorar la salud de sus habitantes (García y García, 2017), lo cual ha llevado a que un $8 \%$ de la población adulta de Estados Unidos (lo que equivale a 18 millones de personas) utilicen algún tipo de meditación como el mindfulness para mejorar su salud (Clarke et al., 2015). Esta información se contrasta con la ausencia de artículos publicados en España, lo cual puede ser un indicador de la falta de expansión de la técnica del mindfulness en este país. Según Mañas (2009) el mindfulness lleva aplicándose hace más de 30 años en Estados Unidos y en España actualmente está en proceso de expansión, lo cual podría explicar la falta de publicaciones en este país. En el caso del resto de países estaríamos en una situación similar a la del ámbito español: Estonia (1), Inglaterra (2), Singapur (1), Corea (1), Sudàfrica (1), Canadà (1) y Australia (1). No podemos realizar 


\section{López Secanell, I.; Gené-Morales, J.}

generalizaciones sobre la expansión del mindfulness a nivel mundial, pero sí que podemos intuir una tendencia a expandirse la técnica más allá del contexto estadounidense.

\section{Nivel educativo, número de participantes y duración}

El número de participantes varia bastante según los estudios analizados. Por una parte, encontramos que la población mínima estudiada es de 12 estudiantes correspondiente al artículo de Khgn (2018). No obstante, es preciso mencionar que este estudio es cualitativo y se basa en el uso de entrevistas. Por otra parte, la población máxima estudiada es la de Nolte et al. (2016) con 342 estudiantes analizados mediante cuestionarios. En general, la población utilizada oscila entre los 15 y 50, aunque hay cuatro estudios que se mueven entre los 80 y los 342 participantes. Estos resultados se deben a dos motivos. El primero porque existen investigaciones cualitativas, las cuales, en general, suelen tener una muestra menor a los estudios cuantitativos. El segundo porque la mayoría de las muestras analizadas se componen de diversos grupos de clase que se comparan con grupos control, lo cual aumenta considerablemente la muestra.

Respecto al nivel educativo, en total encontramos 4 artículos que se aplican con estudiantes de educación primaria frente a 14 aplicados a estudiantes de secundaria. Vemos una predominancia de estudios de secundaria frente a los de primaria, destacando una clara ausencia de trabajos publicados con estudiantes de educación infantil y formación profesional. En el caso de la educación infantil podemos explicar estos resultados por la falta de formación del profesorado en mindfulness (Rechtscgaffen, 2016; López y Beta, 2019) y porque el mindfulness es una técnica que se empieza a aplicar a partir de los 5 años (Rechtscgaffen, 2016), lo cual reduce el ámbito de investigación en esta etapa. En el caso de la formación profesional, consideramos interesante abrir una línea de investigación que nos pueda justificar la falta de estudios e investigaciones en este ámbito.

En relación a la duración de las experiencias, ésta oscila entre 3 y 10 semanas, mientras que excepcionalmente se muestra el trabajo de Hagins y Rundle (2016) con una duración de 11 meses y el trabajo de Meixner et al. (2019) con 20 semanas de duración. En los trabajos de Nolte et al. (2016), Khng (2018), Petterson y Olson (2017) no se especifica la duración, mientras que en el de O'Connor (2019) solamente indica el número de sesiones (12). La media de duración general corresponde con los resultados que se obtienen en los trabajos de Kiens y Larsen (2020), Yook et al. (2017) y Rechtscgaffen (2016) quienes afirman que para ver los efectos del mindfulness es necesario, al menos un trabajo de 8 semanas, ya que es una técnica que requiere constancia y dedicación.

\section{Tipo de investigación}

Los resultados muestran un mayor uso de métodos de investigación cuantitativos (8) que cualitativos (5), aunque la diferencia no es significativa. Según Campayo y Alarcón (2017) una de las principales limitaciones en los estudios en torno el mindfulness es la falta de estudios cualitativos, destacando especialmente aquellos que tratan sobre la formación que tienen los profesores que imparten esta técnica, lo cual no forma parte de ninguno de los objetivos y resultados de los artículos seleccionados. En la metodología cuantitativa los instrumentos de análisis predominantes son los cuestionarios mientras que en los cualitativos destacan la entrevista, las observaciones y análisis de intervenciones. Por una parte, observamos que los métodos cuantitativos se utilizan para investigar la viabilidad, la aceptabilidad, la eficacia y para identificar el efecto de una intervención sobre distintos aspectos medibles relacionados con el bienestar psicológico (la atención, el estrés, la angustia, la concentración, etc.), la autoeficiencia y la autorregulación. Por otra parte, los métodos cualitativos nos acercan a perspectivas más subjetivas que aportan información, principalmente, de las consecuencias de una intervención o la aplicación de un programa de Mindfulness. Tanto las metodologías cuantitativas como cualitativas contribuyen a comprender profundamente las consecuencias a nivel físico, psicológico y emocional de la práctica del mindfulness, ya sea mediante intervenciones o programas.

En el trabajo de O'Connor (2019) también encontramos el análisis del uso de narrativas mediante 12 sesiones de mindfulness en las clases de EF. El uso de las narrativas en la EF es nueva y poco utilizada (Pérez-Samaniego et al., 2011), pero en los últimos años está creciendo su interés en este contexto. Las narrativas permiten crear significados (Bochner, 2002), se adquieren en interacción con otras personas 


\section{Revisión sistemática sobre mindfulness en la EF}

(Stelter, 2006), son personales y sociales (Atkinson, Coffey y Delamont, 2003), representan identidades y nos ayudan a organizar nuestra experiencia temporal (Charon, 2005; Pérez-Samaniego et al., 2011).

Objetivos, resultados y programa mindfulness utilizado

En el análisis de los objetivos y los resultados se observa una gran variedad temática que nos permite evidenciar la diversa aplicabilidad que tiene el mindfulness. La mayoría de los objetivos se centran en el desarrollo de la educación emocional, la atención plena, habilidades de conciencia, la autoeficacia, la autorregulación, la disminución del estrés, la mejora del rendimiento deportivo y la actividad física. También se presentan estudios para analizar la viabilidad de cuestionarios mindfulness como el MAAS (Ullrich-French et al., 2017) y el CAMM (Hagins y Rundle, 2016) para analizar la actividad física de los adolescentes. Respecto al primero de ellos Brown y Ryan (2003) constataron que es una de las escalas más utilizadas en mindfulness y presenta dos adaptaciones, una para adolescentes de 9 a18 años y otra para niños de 8 a 13 años. En cambio, el CAMM es una escala verificada por Greco, Baer y Smith (2011) y es una medida unidimensional que ha sido desarrollado específicamente para medir mindfulness en niños y adolescentes de entre 9 a 18 años.

Por otra parte, también hay objetivos encarados en comprobar la viabilidad de programas de mindfulness como el MBP, el MAC, el MMA, y el MMTS. En relación con estos objetivos se obtienen resultados positivos respecto al uso del mindfulness en el $100 \%$ de los casos. En este sentido, los estudios muestran que el uso de esta técnica dentro de un ámbito deportivo conlleva una mejora de los niveles de atención y autoeficacia de los estudiantes. Respecto al uso de los programas, se constata que el MSPE ayuda a mejorar la ansiedad, la atención plena, la concentración la regulación de las emociones y la satisfacción del rendimiento deportivo. El MBP muestra mejoras en la resiliencia del ego y la regulación emocional. El MAC es eficaz para reducir las dificultades psicológicas y de conducta, la angustia y para mejorar el rendimiento deportivo. Por último, el MMA se evidencia como un programa que promueve la mejora académica, la competencia social, la actividad física, la regulación de las emociones, la confianza en uno mismo, el aumento de la participación y la atención.
A parte de los programas también encontramos estudios centrados en comprobar la viabilidad del uso de cuestionarios mindfulness MS-PA, MAAS y CAMM. El cuestionario MAAS muestra una fiabilidad en la consistencia interna, aunque presenta cierta dificultad para entender algunas de las preguntas de la prueba. Respecto al CAMM los resultados afirman que es una medida apropiada para el desarrollo académico y deportivo de los adolescentes, siempre y cuando tenga una consistencia interna adecuada. Finalmente, el MS-PA muestra indicios de buena fiabilidad y consistencia, aunque se requieren futuros estudios para ello.

En cuanto a los programas de mindfulness utilizados observamos que, de los 15 artículos, 9 no han utilizado ningún programa, sino que se han basado en intervenciones o en el uso de cuestionarios. Es preciso destacar la relación de estos estudios con sus países de origen ya que, de los 15 estudios, Canadá (1) y Singapur (1) y Estados Unidos (4) son los únicos países que utilizan programas de mindfulness, lo cual se relaciona con la extensión de esta técnica en América. Se constata como los programas más utilizados son MBP (1), MAC (2), MMA (2), y MSPE (2), MMTS (1), sin aportar un resultado concluyente sobre la predominancia de un tipo de programa respecto a otro. Como se indicó en el estudio de García et al. (2017) la implementación de programas basados en la atención plena en las escuelas se presenta como una vía de ayuda para niños y adolescentes respecto a su autorregulación emocional y atencional, favoreciendo su adaptación al contexto y para alcanzar un mayor bienestar psicológico.

\section{CONCLUSIONES}

El objetivo de este trabajo era realizar un análisis sistemático de los artículos publicados en los últimos seis años sobre el uso de mindfulness en la EF de los distintos niveles educativos (educación infantil, primaria, secundaria y formación profesional), así como sus principales conclusiones.

La revisión realizada se ha basado en 15 artículos en los últimos seis años, lo cual es una evidencia de que, aunque en los últimos años se ha incrementado el interés por el mindfulness y se han demostrado sus beneficios, generalmente aun son pocas las intervenciones realizadas en la EF en el mundo. De estos 15 artículos, ninguno se ha realizado en España, 


\section{López Secanell, I.; Gené-Morales, J.}

cumpliéndose así la hipótesis planteada en esta investigación. Este aspecto es revelador por dos motivos. El primero porque se evidencia como en el estado español la técnica de mindfulness aun está poco implementada en el ámbito de la EF, aun los numerosos estudios que muestras los beneficios del uso de esta técnica en el deporte. Entre ellos encontramos el trabajo de Lu, Tito y Kentel (2009) quienes defienden la necesidad de extender el uso del mindfulness en la EF porque aporta consciencia y una perspectiva holística de entender el cuerpo, lo cual va más allá del trabajo puramente físico. El segundo, porque se muestra cierta distancia entre los estudios teóricos que demuestran los numerosos beneficios de esta técnica en el mundo del deporte y las prácticas que se llevan a cabo en los contextos escolares. Este resultado es importante para el profesorado de EF, ya que muestra la necesidad de seguir aplicando intervenciones de mindfulness en esta materia para ver sus posibilidades de uso.

Los resultados también muestran que los estudios analizados se aplican principalmente en la educación secundaria. Sería necesario investigar la falta de investigaciones en educación infantil, primaria y formación profesional, ya que las investigaciones nos afirman que el mindfulness puede empezar a aplicarse a partir de los 5 años (Rechtscgaffen, 2016). Puede que la falta de formación del profesorado en mindfulness sea una de las posibles causas de esta, ya que, según si queremos fomentar el uso de esta técnica dentro de la materia de $\mathrm{EF}$, primero será necesario formar al profesorado para que éste lo incorpore en su vida y, consecuentemente, en su contexto laboral (López y Beta, 2019).

El trabajo realizado nos aporta una revisión completa, concisa, objetiva y lógica de las investigaciones sobre mindfulness que se han implementado en contextos educativos y, concretamente, en el ámbito de la EF. Este tipo de investigación representa una novedad, ya que no existen revisiones sistemáticas sobre los últimos seis años sobre la relación entre el mindfulness y la EF. No obstante, se presentan unas limitaciones que especificamos a continuación. Una primera limitación es considerar solamente publicaciones en inglés y castellano en los criterios de inclusión, ya que se podrían considerar otras como el alemán o el portugués. Otra limitación es la falta de análisis de la muestra en relación a su género, así como el procedimiento de revisión. En este último caso se ha intentado minimizar las posibilidades de sesgo partiendo del planteamiento de la investigación a partir de una adaptación de otro trabajo publicado (Barba et al., 2020) y mediante la participación de dos colaboradores externos expertos en psicología y deporte para analizar grado de inclusión en JCR o SJR, el detalle del proceso metodológico utilizado, el número de participantes, la duración y el nivel de calidad JCR de los artículos.

Para futuras líneas de investigación se propone la realización de un meta-análisis para responder preguntas como: ¿Son similares los resultados obtenidos en los diferentes estudios? ¿En caso de que las conclusiones sean similares se podrían plantear cuestiones como "cuál es la estimación general del efecto?" ¿Qué tan precisa y sólida es esta estimación? ¿Se pueden explicar las diferencias?" También se propone el diseño de un programa especifico de mindfulness para la EF adaptable a los distintos niveles educativos y que pueda ser aplicado en los centros. A partir de este diseño se puede plantear un análisis cuantitativo y cualitativo de las consecuencias de la implementación del programa en el bienestar físico, psicológico y emocional de los estudiantes.

\section{APLICACIONES PRÁCTICAS}

- Tener un marco teórico actualizado y de referencia para diseñar programas o intervenciones prácticas de mindfulness en contextos educativos deportivos.

- Identificar la aplicabilidad de la evidencia conocida sobre mindfulness en el contexto de la EF.

- Usar la revisión bibliográfica para explicar, apoyar o ampliar la teoría generada en estudios de investigación sobre mindfulness y deportes.

\section{REFERENCIAS}

1. Anand, U. y Sharma, M. P (2014) Effectiveness of a mindfulness-based stress reduction program on stress and well-being in adolescents in a school setting. Indian Journal of Positive Psychology, 5(1), 17.

2. Atkinson, P., Coffey, A. y Delamont, S. (2003). Key themes in qualitative research. Altamira.

3. Ato, M., López, J. J. y Benavente, A. (2013). Un 


\section{Revisión sistemática sobre mindfulness en la EF}

sistema de clasificación de los diseños de investigación en psicología. Anales de Psicología, 29(3), 1038-1059. https://doi.org/10.6018/analesps.29.3.178511

4. Barba, R. A., Bores, D., Hortigüela, D., y González-, G. (2020). The application of the teaching games for understanding in physical education. systematic review of the last six years. International Journal of Environmental Research and Public Health, 17(3330), 1-16. https://doi.org/10.3390/ijerph17093330

5. Beltrán, M. J. C., y Abad, P. (2011). La respiración consciente como factor principal de la relajación en la educación física escolar. EmásF: Revista Digital de Educación Física, 13, 19-31. https://doi.org/10.28997/ruefd.v0i11.1

6. Biegel, G. y Brown, K. W. (2010). Assessing the efficacy of an adapted in class mindfulness-based training program for school-age children: a pilot study. Recuperado de https://goo.gl/3rnRd1

7. Bochner, A. (2002). Perspectives on inquiry III: The moral of stories. In: KNAPP, M.; Daley, J. (Eds.), The handbook of interpersonal communication. Sage.

8. Britton, W. B., Lepp, N. E., Niles, H. F., Rocha, T., Fisher, N. E. y Gold, J. S. A. (2014). Randomized controlled pilot trial or classroom base mindfulness meditation compared to an active control condition in sixth-grade children. Journal of School Psychology, 52(3), 263-278. https://doi.org/10.1016/j.jsp.2014.03.002

9. Broderick, P. C. y Metz, S. (2009). Learning to Breathe: A pilot trial of mindfulness curriculum for adolescents. Advances in School Mental Health Promotion, 2(1), 35-46. https://doi.org/10.1080/1754730X.2009.9715696

10. Brown, K. W., y Ryan, R. M. (2003). The benefits of being present: mindfulness and its role in phychological well-being. Journal of personality and social psychology, 84(4), 822-848. https://doi.org/10.1037/0022-3514.84.4.822

11. Campayo, J., y Alarcón, M. (2017). Evidencias científicas de mindfulness aplicadas a la educación. Desarrollando la atención plena en las aulas. En J. Campayo (Presidencia), International Meeting on Mindfulness. Congreso llevado a cabo en Zaragoza, España.

12. Charon R. (2005). A narrative medicine for pain. En D. Carr, J. Loeser y D. Morris (Eds.) Narrative, pain and suffering. (pp. 29-44). IASP.
13. Clarke, T. C., Black, L. I., Stussman, B. J., Barnes, P. M., y Nahin, R. L. (2015). Trends in the use of complementary health approaches among adults: United States, 2002-2012. National Health Statistics Reports, 79, 1-16.

14. Cox, A. E., Ullrich-French, S., y French, B. F. (2016). Validity evidence for the state mindfulness scale for physical activity. Measurement in Physical Education and Exercise $\quad$ Science, 20(1), 38-49. https://doi.org/10.1080/1091367X.2015.1089404

15. Del Águila, J., Mañas, I., Clemente, F., Gil, C. y Montoya, M. (2009). Programas basados en mindfulness para atletas. En A. J. Cangas, J. Gallego y N. Navarro (Eds.), Actividad física y deporte adaptado (pp. 149-158). Alborán Editores.

16. Delgado, L. C. (2009). Correlatos psicofisiológicos de mindfulness y la preocupación. Eficacia de un entrenamiento de habilidades mindfulness. (Tesis doctoral, Universidad de Granada, Granada, España). Recuperado de https://goo.gl/EruDyu

17. Desmond, C. T., Hanich, L. y Millersville, P. A. (2010). The effects of mindful awareness teaching practices on the executive functions of students in an urban, low-income middle school. Millerville University.

18. Dunn, B. R., Hartigan, J. A. y Mikulas, W. L. (1999). Concentration and mindfulness meditations: unique forms of consciousness? Applied Psychophysiology and Biofeedback, 24(3), 147-165. https://doi.org/10.1023/A:1023498629385

19. Ergas, O. y Hadar, L. L. (2019). Mindfulness in and as education: A map of a developing academic discourse from 2002 to 2017. Review of Education, 7(3), 757-797. https://doi.org/10.1002/rev3.3169

20. Firth-Clark, A., Sütterlin, S. y Lugo, R. G. (2019). Using cognitive behavioral techniques to improve academic achievement in student-athletes, Education Sciences, 9(2), 1-16. https://doi.org/10.3390/educsci9020089

21. Flook, L., Smalley, S. L., Kitil, M. J., Galla, B. M., Kaiser-Greenland, S., Locke, J., Ishijima, E. y Kasari, C. (2010). Effects of mindful awareness practices on executive functions in elementary school children. Journal of Applied School $\begin{array}{lll}\text { Psychology, } & \text { 26(1), }\end{array}$ 


\section{López Secanell, I.; Gené-Morales, J.}

https://doi.org/10.1080/15377900903379125

22. Franco, C. (2009). Modificación de los niveles de burnout y de personalidad resistente en un grupo de deportistas a través de un programa de conciencia plena. Anuario de Psicología, 40, 377390. https://doi.org/10.1016/j.aprim.2009.10.020

23. Garcés, E. J., De Francisco, C., y Arce, C. (2012). Inventario de burnout en deportistas revisado (IBD-R). Revista de Psicología del Deporte, 21(2), 271-278. https://doi.org/10.4321/s1578$\underline{84232014000100004}$

24. García, J., Demarzo, M y Modregó, M. (2017). Bienestar emocional y midfulness en la educación. Alianza Editorial.

25. García, R. R. T., y García-Campayo, J. (2017). El impacto de mindfulness en el mundo. Recuperado de http://menteserena.mx/el-impacto-demindfulness-en-el-mundo/

26. Gardner, F. L. y Moore, Z. E. (2007). The psychology of enhancing human performance: The Mindfulness-Acceptance-Commitment (MAC) approach. New York: Springer Publishing Company.

27. Gardner, F. L. y Moore, Z. E. (2004). A mindfulness-acceptance-commitment-based approach to athletic performance enhancement: theoretical considerations. Beaver Therapy, 35(4), 707-723. https://doi.org/10.1016/S0005-7894(04)80016-9

28. Gooding, A. y Gardner, F. L. (2009). An investigation of the relationship between mindfulness, preshot routine, and basketball free throw percentage. Journal of Clinical Sport Psychology, 3(4), 303-319. https://doi.org/10.1123/jcsp.3.4.303

29. Greco, L. A., Baer, R. A. y Smith, G. T. (2011). Assessing mindfulness in children and adolescents: development and validation of the Child and Adolescent Mindfulness Measure (CAMM). Psychological Assessment, 23(3), 606614. https://doi.org/10.1037/a0022819

30. Gross, M., Moore, Z. E., Gardner, F. L., Wolanin, A. T., Pess, R. y Marks, D. R. (2016). An empirical examination comparing the Mindfulness-Acceptance- Commitment approach and Psychological Skills Training for the mental health and sport performance of female student athletes. International Journal of Sport and Exercise Psychology, 16(4), 431-451.
https://doi.org/10.1080/1612197X.2016.1250802

31. Hagins, M., y Rundle, A. (2016). Yoga improves academic performance in urban high school students compared to physical education: a randomized controlled trial. Mind, Brain, and Education, 10(2), https://doi.org/10.1111/mbe. 12107

105-116.

32. Hasker, S. M. (2010). Evaluation of the Mindfulness-Acceptance-Commitment (MAC) approach for enhancing athletic performance. Dissertation Abstracts International, 71(9-B), pp. 57-90.

33. Kabat-Zinn, J. (2003). Mindfulness based intervention in context: past, present and future. Clinical Psychology: Science and Practice, 10(2), 144-146. https://doi.org/10.1093/clipsy.bpg016

34. Kauffman, K. A., Glass, C. R. y Arnkoff, D. B. (2009). Evaluation of Mindful Sport Performance Enhancement (MSPE): a new approach to promote flow in athletes. Journal of Clinical Sports Psychology, 4, 334-356. https://doi.org/10.1123/jcsp.3.4.334

35. Khng, K. H. (2018). Mindfulness in education: the case of Singapore, Learning: Research and Practice, 4(1), 52-65. https://doi.org/10.1080/23735082.2018.1428120

36. Kiens, K., y Larsen, C. H. (2020). Provision of a mental skills intervention program in an elite sport school for student-athletes. Journal of Sport Psychology in Action, 1-15. https://doi.org/10.1080/21520704.2020.1765925

37. Kuyken, W., Weare, K., Ukoumunne, C., Vicary, R., Motton, N., Burnett, R., Cullen, C., Hennelly, S. y Huppert, F. (2013). Effectiveness of the mindfulness in schools programme: nonrandomised controlled feasibility study. The British Journal of Psychiatry, 203(2), 126-131. https://doi.org/10.1192/bjp.bp.113.1266

38. López, I. y Beta, M. (2019). "Mindfulness" y educación: formación de los instructores de "mindfulness" en Educación Secundaria. Didacticae, 6, 125-143. https://doi.org/10.1344/did.2019.6.126-143

39. Lu, C., Tito, J. M., y Kentel, J. A. (2009). Eastern movement disciplines (EMDs) and mindfulness: A new path to subjective knowledge in Western physical education. Quest, 61(3), 353-370. https://doi.org/10.1080/00336297.2009.1048362 1 


\section{Revisión sistemática sobre mindfulness en la EF}

40. Luiselli, J. K., Worthen, D., Carbonell, L., y Queen, A. H. (2017). Social validity assessment of mindfulness education and practices among high school students. Journal of applied school psychology, 33(2),

124-135. https://doi.org/10.1080/15377903.2016.1264531

41. Lutkenhouse, J. M. (2007). The case of Jenny: A freshman collegiate athlete experiencing performance dysfunction. Journal of Clinical Sport Psychology, 1, 166-180. https://doi.org/10.1123/jcsp.1.2.166

42. Lutkenhouse, J., Gardner, F. L. y Morrow, C. (2007). A randomized controlled trial comparing the performance enhancement effects of Mindfulness-Acceptance-Commitment (MAC) performance enhancement and psychological skills training procedures. Manuscript in preparation.

43. Mañas, I. (2009). Mindfulness (Atención Plena): La meditación en psicología clínica. Gaceta de Psicología, 50, 13-29.

44. Meixner, T., Irwin, A., Miscio, M. W., Cox, M., Woon, S., McKeough, T. y Milligan, K. (2019). Delivery of integra mindfulness martial arts in the secondary school setting: factors that support successful implementation and strategies for navigating implementation challenges. School Mental Health, 11(3), 549-561. https://doi.org/10.1007/s12310-018-9301-4

45. Metz, S. M., Frank, J. L., Reibel, D., Cantrell, T., Sanders, R. y Broderick, P. C. (2013). The effectiveness of the learning to Breathe program on adolescent emotion regulation. Research in Human Development, 10(3), 252-272. https://doi.org/10.1080/15427609.2013.818488

46. Milligan, K., Cosme, R., Miscio, M. W., Mintz, L., Hamilton, L., Cox, M., Woon, S., Gage, M. y Phillips, M. (2017). Integrating mindfulness into mixed martial arts training to enhance academic, social, and emotional outcomes for at-risk high school students: A qualitative exploration. Contemporary School Psychology, 21(4), 335-346. https://doi.org/10.1007/s40688-017-0142-1

47. Minkler, T. O., Glass, C. R. y Hut, M. (2020). Mindfulness training for a college team: Feasibility, acceptability, and effectiveness from within an athletic department. Journal of Applied Sport Psychology, 1-18. https://doi.org/10.1080/10413200.2020.1739169
48. Mulhearn, S. C., Kulinna, P. H. y Lorenz, K. A. (2017). Harvesting harmony: Mindfulness in physical education. Journal of Physical Education, Recreation y Dance, 88(6), 44-50. https://doi.org/10.1080/07303084.2017.1330168

49. Napoli, M., Krech, P. R. y Holley, L. C. (2005). Mindfulness training for elementary school students: The attention academy. Journal of Applied School Psychology, 21(1), 99-125. https://doi.org/10.1300/J370v21n01_05

50. Nolte, K., Steyn, B. J., Krüger, P. E. y Fletcher, L. (2016). Mindfulness, psychological well-being and doping in talented young high-school athletes. South African Journal for Research in Sport, Physical Education and Recreation, 38(2), 153-165.

51. O'Connor, J. (2019). Exploring a pedagogy for meaning-making in physical education. European Physical Education Review, 25(4), 1093-1109. https://doi.org/10.1177/1356336X18802286

52. Palmi, J. y Solé, S. (2016). Intervenciones basadas en Mindfulness (Atención Plena) en Psicología del Deporte. Revista de Psicología del Deporte, 25 , 147-155. https://doi.org/10.5093/rpadef2018a14

53. Pérez-Samaniego, V. M., Devís-Devís, J., Smith, B. M., y Sparkes, A. C. (2011). La investigación narrativa en la educación física y el deporte: qué es y para qué sirve. Movimento, 17(1), 11-38. https://doi.org/10.22456/1982-8918.71277

54. Pérez, Mª . A. y Botella, L. (2006). Conciencia plena (mindfulness) y psicoterapia: concepto, evaluación y aplicaciones clínicas. Revista de Psicoterapia, 7(66), 77-120.

55. Petterson, H., y Olson, B. L. (2017). Effects of mindfulness-based interventions in high school and college athletes for reducing stress and injury and improving quality of life. Journal of sport rehabilitation, 26(6),

578-587. https://doi.org/10.1123/jsr.2016-0047

56. Potek, R. (2012). Mindfulness as a School: Based Prevention Program and its Effect on Adolescent Stress, Anxiety and Emotion Regulation. New York University.

57. Rechtscgaffen, D. J. (2016) Educación mindfulness. El cultivo de la consciencia y la atención para profesores y alumnos. Gaia Ediciones.

58. Rosaen, C. y Benn, R. (2006). The experience of transcendental meditation in middle school 


\section{López Secanell, I.; Gené-Morales, J.}

students: a qualitative report. Explore: The Journal of Science and Healing, 2, 422-425. https://doi.org/10.1016/j.explore.2006.06.001

59. Saltzman, A. y Goldin, P. (2008). Mindfulness based stress reduction for school-age children, En S. C. Hayes y L. A. Greco (eds). Acceptance and mindfulness interventions for children adolescents and families (pp.139-161). New Harbinger.

60. Schonert-Reichl, E. y Lawlor, M.S. (2010). The effects of a mindfulness-based educaion progam on pre-and early adolescents' well-being and social and emotional competence. Mindfulness, l(3), 137-151. https://doi.org/10.1007/s12671010-0011-8

61. Schonert-Reichl, E., Oberle, E., Lawlor, M. S., Abbott, D., Thomson, K., Oberlander, T. F. y Diamond, A. (2015). Enhancing cognitive and social-emotional development through a simpleto-administer mindfulness-based school program for elementary school children: a randomized controlled trial. Psychology, 51, 52-56. https://doi.org/10.1037/a0038454

62. Schwanhausser, L. (2009). Application of the Mindfulness-Acceptance-Commitment (MAC) Protocol with an adolescent springboard diver: the case of Steve. Journal of Clinical Sports Psychology, 3, 377-396. https://doi.org/10.1123/jesp.3.4.377

63. So, K. T y Orme-Johnson, D. W. (2001). Three randomized experiments on the longitudinal effects of the Transcedental Meditation technique on cognition, Intelligence, 29(5), 419-440. https://doi.org/10.1016/S0160-2896(01)00070-8

64. Solé, S., Carrança, B., Serpa, S. y Palmi, J. (2014). Aplicaciones del mindfulness (conciencia plena) en lesión deportiva. Revista de Psicología del Deporte, 23, 501-508.

65. Stelter, R. (2007). Coaching: a process of personal and social meaning making. International Coaching Psychology Review, 2, 191-201.

66. Tang, Y. Y., Yang, L., Leve, L. D. y Harold, G. T. (2012). Improving executive function and its neurobiological mechanisms through a mindfulness-based intervention: Advances within the field of developmental neuroscience. Child Development Perspectives, 6, 361-366. https://doi.org/10.1111/j.17508606.2012.00250.x

67. Ullrich-French, S., Cox, A., Cole, A., Rhoades Cooper, B. y Gotch, C. (2017). Initial validity evidence for the state mindfulness scale for physical activity with youth. Measurement in Physical Education and Exercise Science, 21(4), 177-189.

https://doi.org/10.1080/1091367X.2017.1321543

68. Urrútia, G. y Bonfill, X. (2010). Declaración PRISMA: una propuesta para mejorar la publicación de revisiones sistemáticas y metaanálisis. Medicina Clínica, 135(11), 507-511. https://doi.org/10.1016/j.medcli.2010.01.015

69. Wolanin, A. T. (2005). Mindfulness-AcceptanceCommitment (MAC) based performance enhancement for Division I collegiate athletes: A preliminary investigation (Doctoral dissertation, La Salle University, 2003). Dissertation Abstracts International-B, 65, 3735-3794.

70. Worthen, D. y Luiselli, J. K. (2016). Attitudes and opinions of female high school athletes about sports-focused mindfulness training and practices, Journal of Clinical Sport Psychology, 10(3), pp. 177-191. https://doi.org/10.1123/jcsp.2016-0005

71. Yook, Y.-S., Kang, S.-J. y Park, I. (2017). Effects of physical activity intervention combining a new sport and mindfulness yoga on psychological characteristics in adolescents, International Journal of Sport and Exercise Psychology, 15(2), 109-117. https://doi.org/10.1080/1612197X.2015.1069878 


\section{Revisión sistemática sobre mindfulness en la EF}

\begin{tabular}{|c|c|c|c|c|c|c|c|c|c|}
\hline $\begin{array}{c}\text { Autor y } \\
\text { año }\end{array}$ & $\begin{array}{l}\text { País e } \\
\text { idioma }\end{array}$ & $\begin{array}{l}\text { Nivel educativo } \\
\text { y no de } \\
\text { participantes }\end{array}$ & Duración & $\begin{array}{c}\text { Tipo } \\
\text { investigación }\end{array}$ & Objetivos & Medidas & Resultados & $\begin{array}{l}\text { Programa de } \\
\text { mindfulness } \\
\text { utilizado }\end{array}$ & $\begin{array}{c}\text { d/medidas } \\
\text { pre y post- } \\
\text { intervención }\end{array}$ \\
\hline $\begin{array}{l}\text { Kiens y } \\
\text { Larsen } \\
(2020)\end{array}$ & $\begin{array}{l}\text { Estonia. } \\
\text { Inglés }\end{array}$ & $\begin{array}{l}\text { Educación } \\
\text { secundaria ( } 39 \\
\text { estudiantes) }\end{array}$ & $\begin{array}{l}8 \text { semanas } \\
\text { (16 sesiones) }\end{array}$ & $\begin{array}{l}\text { Cualitativo: } \\
\text { observación y } \\
\text { comunicación. } \\
\text { Cuestionario } \\
\text { anónimo y } \\
\text { voluntario con } \\
\text { feedback. }\end{array}$ & $\begin{array}{l}\text { Desarrollar la capacidad de } \\
\text { atención plena. Introducir } \\
\text { ejercicios de atención plena } \\
\text { como una forma de entrenar } \\
\text { las habilidades de atención y } \\
\text { fortalecer las habilidades de } \\
\text { conciencia y atención } \\
\text { aprendidas a través de la } \\
\text { práctica. }\end{array}$ & $\begin{array}{l}\text { 1. Autoanálisis } \\
\text { 2. Autoconocimiento } \\
\text { 3. Aceptación } \\
\text { 4. Dedicación } \\
\text { 5. Control de la } \\
\text { atención }\end{array}$ & $\begin{array}{l}\text { Los estudiantes mostraron } \\
\text { una buena actitud al } \\
\text { estudio realizado. Con las } \\
\text { indicaciones dadas } \\
\text { pudieron conseguir los } \\
\text { objetivos mencionados. }\end{array}$ & - & - \\
\hline $\begin{array}{l}\text { Minkler } \\
\text { et al. } \\
(2020)\end{array}$ & $\begin{array}{l}\text { Estados } \\
\text { Unidos. } \\
\text { Inglés }\end{array}$ & $\begin{array}{l}\text { Bachillerato ( } 30 \\
\text { estudiantes) }\end{array}$ & $\begin{array}{l}6 \text { semanas (6 } \\
\text { sesiones) }\end{array}$ & $\begin{array}{l}\text { Cuantitativo: } \\
\text { cuestionarios }\end{array}$ & $\begin{array}{l}\text { Investigar la viabilidad, } \\
\text { aceptabilidad y eficacia de } \\
\text { la metodología aplicada. }\end{array}$ & $\begin{array}{l}\text { 1. "Flow" deportivo } \\
\text { 2. Ansiedad deportiva } \\
\text { 3. Mindfulness } \\
\text { 4. Mindfulness } \\
\text { deportivo } \\
\text { 5. Dificultad en } \\
\text { regulación de } \\
\text { emociones } \\
\text { 6. Autopercepción de } \\
\text { rendimiento } \\
\text { deportivo } \\
\text { 7. Percepción del } \\
\text { entrenador del } \\
\text { rendimiento } \\
\text { deportivo }\end{array}$ & $\begin{array}{l}\text { Mejoras significativas en } \\
\text { las medidas de ansiedad, } \\
\text { atención plena, } \\
\text { dificultades en la } \\
\text { regulación de las } \\
\text { emociones y satisfacción } \\
\text { del rendimiento deportivo. }\end{array}$ & MSPE & $\begin{array}{l}\text { 1. } d=.55 \\
\text { 2. } d=1.22 \\
\text { 3. } d=.74 \\
\text { 4. } d=.46 \\
\text { 5. } d=1.19 \\
\text { 6. } d=.91 \\
\text { 7. } d=2.00\end{array}$ \\
\hline $\begin{array}{l}\text { Firth- } \\
\text { Clark et } \\
\text { al. (2019) }\end{array}$ & $\begin{array}{l}\text { Inglaterra. } \\
\text { Inglés }\end{array}$ & $\begin{array}{l}\text { Bachillerato (94 } \\
\text { estudiantes) }\end{array}$ & $\begin{array}{l}6 \text { semanas } \\
\text { (10 sesiones) }\end{array}$ & $\begin{array}{l}\text { Cuantitativo: } \\
\text { intervenciones }\end{array}$ & $\begin{array}{l}\text { Analizar la función de la } \\
\text { autoeficacia generalizada y } \\
\text { la autorregulación. } \\
\text { Examinar la autorregulación } \\
\text { en términos de permitir al } \\
\text { estudiante aumentar los } \\
\text { sentimientos positivos sobre } \\
\text { qué tan bien puede controlar } \\
\text { sus experiencias de } \\
\text { aprendizaje. }\end{array}$ & $\begin{array}{l}\text { 1. Autorregulación } \\
\text { 2. Autoeficacia } \\
\text { 3. Rendimiento } \\
\text { académico }\end{array}$ & $\begin{array}{l}\text { Se encontraron diferencias } \\
\text { significativas antes y } \\
\text { después de la intervención } \\
\text { en todo el proceso y la } \\
\text { autoeficacia generalizada } \\
\text { por el mindfulness. }\end{array}$ & $\begin{array}{l}\text { Psicología deportiva: } \\
\text { autodiálogo, } \\
\text { visualizar, etc.) }\end{array}$ & $\begin{array}{l}\text { 1. } d=.41 \\
\text { 2. } d=.81 \\
\text { 3. } d=1.53\end{array}$ \\
\hline
\end{tabular}




\section{López Secanell, I.; Gené-Morales, J.}

\begin{tabular}{|c|c|c|c|c|c|c|c|c|c|}
\hline $\begin{array}{l}\text { Khng } \\
(2018)\end{array}$ & $\begin{array}{l}\text { Singapur. } \\
\text { Inglés }\end{array}$ & $\begin{array}{l}\text { Educación } \\
\text { Primaria y } \\
\text { Secundaria }\end{array}$ & $\begin{array}{l}\text { Artículo de } \\
\text { revisión }\end{array}$ & $\begin{array}{l}\text { Cualitativo: } \\
\text { entrevistas y } \\
\text { revisión }\end{array}$ & $\begin{array}{l}\text { Analizar la forma en la cual los } \\
\text { MBP se incorporan al entorno } \\
\text { escolar }\end{array}$ & - & $\begin{array}{l}\text { Los resultados muestran } \\
\text { mejoras en mindfulness, la } \\
\text { resiliencia del ego y la } \\
\text { regulación emocional. }\end{array}$ & MBP & - \\
\hline $\begin{array}{l}\text { Petterson } \\
\text { y Olson } \\
\text { (2017) }\end{array}$ & $\begin{array}{l}\text { Estados } \\
\text { Unidos. } \\
\text { Inglés }\end{array}$ & $\begin{array}{l}\text { Educación } \\
\text { Secundaria y } \\
\text { Bachillerato } \\
\text { (23 estudiantes) }\end{array}$ & - & $\begin{array}{l}\text { Cualitativo: } \\
\text { Intervenciones }\end{array}$ & $\begin{array}{l}\text { Utilizar intervenciones basadas } \\
\text { en mindfulness para reducir el } \\
\text { estrés y las lesiones, y mejorar } \\
\text { la calidad de vida general de los } \\
\text { estudiantes. }\end{array}$ & $\begin{array}{l}\text { 1. Estrés } \\
\text { 2. Lesiones } \\
\text { 3. Calidad de vida }\end{array}$ & $\begin{array}{l}\text { Incremento de la atención } \\
\text { y control de las emociones } \\
\text { negativas y el estrés. }\end{array}$ & - & - \\
\hline $\begin{array}{l}\text { Yook et } \\
\text { al. (2017) }\end{array}$ & $\begin{array}{l}\text { Corea. } \\
\text { Inglés }\end{array}$ & $\begin{array}{l}\text { Educación } \\
\text { Primaria (46 } \\
\text { estudiantes) }\end{array}$ & $\begin{array}{l}8 \text { semanas ( } 8 \\
\text { sesiones) }\end{array}$ & $\begin{array}{l}\text { Cuantitativo: } \\
\text { cuestionarios }\end{array}$ & $\begin{array}{l}\text { Identificar el efecto de una } \\
\text { intervención de actividad física } \\
\text { que combina un nuevo deporte } \\
\text { y el yoga sobre las } \\
\text { características psicológicas de } \\
\text { los adolescentes. }\end{array}$ & $\begin{array}{l}\text { 1. Autoestima } \\
\text { 2. Resiliencia } \\
\text { 3. Felicidad }\end{array}$ & $\begin{array}{l}\text { En el grupo experimental, } \\
\text { la autoestima de los } \\
\text { participantes del estudio, } \\
\text { la resiliencia y la felicidad } \\
\text { cambiaron } \\
\text { significativamente durante } \\
\text { el proceso. }\end{array}$ & - & $\begin{array}{l}\text { 1. } d=.56 \\
\text { 2. } d=.94 \\
\text { 3. } d=.70\end{array}$ \\
\hline $\begin{array}{l}\text { Worthen } \\
\text { y Luiselli } \\
\text { (2016) }\end{array}$ & $\begin{array}{l}\text { Estados } \\
\text { Unidos. } \\
\text { Inglés }\end{array}$ & $\begin{array}{l}\text { Bachillerato (32 } \\
\text { estudiantes) }\end{array}$ & $\begin{array}{l}10 \text { semanas } \\
\text { (10 sesiones) }\end{array}$ & $\begin{array}{l}\text { Cuantitativo: } \\
\text { cuestionarios }\end{array}$ & $\begin{array}{l}\text { Ilustrar el proceso de validación } \\
\text { social del mindfulness y las } \\
\text { prácticas basadas en el mismo. }\end{array}$ & $\begin{array}{l}\text { 1. Conciencia } \\
\text { emocional } \\
\text { 2. Foco atencional }\end{array}$ & $\begin{array}{l}\text { El mindfulness tiene } \\
\text { efectos positivos en la } \\
\text { conciencia emocional y la } \\
\text { concentración. }\end{array}$ & MAC/MMTS/MSPE & - \\
\hline $\begin{array}{l}\text { Nolte et } \\
\text { al. (2016) }\end{array}$ & $\begin{array}{l}\text { Sudáfrica. } \\
\text { Inglés }\end{array}$ & $\begin{array}{l}\text { Educación } \\
\text { Secundaria ( } 342 \\
\text { estudiantes) }\end{array}$ & - & $\begin{array}{l}\text { Cuantitativo: } \\
\text { cuestionarios }\end{array}$ & $\begin{array}{l}\text { Determinar cómo el } \\
\text { mindfulness y el bienestar } \\
\text { psicológico se relacionan con la } \\
\text { propensión a utilizar fármacos } \\
\text { para mejorar el rendimiento en } \\
\text { una muestra estudiantes. }\end{array}$ & $\begin{array}{l}\text { Correlaciones entre } \\
\text { propensión a utilizar } \\
\text { fármacos y los } \\
\text { constructos: } \\
\text { 1. Mindfulness } \\
\text { 2. Bienestar } \\
\text { psicológico }\end{array}$ & $\begin{array}{l}\text { Mindfulness y el } \\
\text { entrenamiento de } \\
\text { habilidades psicológicas } \\
\text { pueden ser intervenciones } \\
\text { apropiadas para que se } \\
\text { disminuya la probabilidad } \\
\text { del uso de fármacos, así } \\
\text { como para mejorar su } \\
\text { rendimiento deportivo. }\end{array}$ & - & $\begin{array}{l}1 . r=-.08 \\
\text { de }-.19 \text { a } .06 \\
2 . r=.00 \\
\text { de }-.20 \text { a } .16\end{array}$ \\
\hline $\begin{array}{l}\text { Gross et } \\
\text { al. (2016) }\end{array}$ & $\begin{array}{l}\text { Estados } \\
\text { Unidos. } \\
\text { Inglés }\end{array}$ & $\begin{array}{l}\text { Bachillerato ( } 22 \\
\text { estudiantes) }\end{array}$ & $\begin{array}{l}7 \text { semanas ( } 7 \\
\text { sesiones) }\end{array}$ & $\begin{array}{l}\text { Cuantitativo: } \\
\text { cuestionarios }\end{array}$ & $\begin{array}{l}\text { Examinar la eficacia del } \\
\text { programa MAC para las } \\
\text { dificultades de } \\
\text { comportamiento, la angustia } \\
\text { emocional, las dificultades } \\
\text { psicológicas y el rendimiento } \\
\text { deportivo en comparación con } \\
\text { un tratamiento activo. }\end{array}$ & $\begin{array}{l}\text { 1. Síntomas } \\
\text { psicológicos } \\
\text { 2. Flexibilidad } \\
\text { psicológica } \\
\text { 3. Dificultad en la } \\
\text { regulación de } \\
\text { emociones } \\
\text { 4. Mindfulness } \\
\text { 5. Percepción del } \\
\text { entrenador del } \\
\text { rendimiento deportivo } \\
\text { 6. Deseabilidad social } \\
\text { 7. Alianza de trabajo }\end{array}$ & $\begin{array}{l}\text { El MAC es eficaz para } \\
\text { reducir las dificultades } \\
\text { psicológicas y de } \\
\text { conducta, la angustia } \\
\text { emocional al tiempo que } \\
\text { mejora el rendimiento } \\
\text { deportivo. }\end{array}$ & MAC & $\begin{array}{l}1 . d=.84 \\
\text { de } .20 \text { a } 1.16 \\
\text { 2. } d=1.47 \\
\text { 3. } d=.74 \\
\text { 4. } d=.35 \\
\text { 5. } d=1.09 \\
\text { 6. }- \\
\text { 7. } d=0.12\end{array}$ \\
\hline
\end{tabular}




\section{Revisión sistemática sobre mindfulness en la EF}

\begin{tabular}{|c|c|c|c|c|c|c|c|c|c|}
\hline $\begin{array}{l}\text { Milligan } \\
\text { et al. } \\
(2017)\end{array}$ & $\begin{array}{l}\text { Canadá. } \\
\text { Inglés }\end{array}$ & $\begin{array}{l}\text { Educación } \\
\text { Secundaria y } \\
\text { Bachillerato } \\
\text { (41 } \\
\text { estudiantes) }\end{array}$ & $\begin{array}{l}20 \\
\text { semanas } \\
(20 \\
\text { sesiones })\end{array}$ & $\begin{array}{l}\text { Cualitativo: } \\
\text { entrevistas }\end{array}$ & $\begin{array}{l}\text { Evaluar la efectividad de } \\
\text { la implementación de } \\
\text { MMA en los estudiantes. }\end{array}$ & $\begin{array}{l}\text { 1. Percepción sobre el } \\
\text { programa } \\
\text { 2. Cambios auto- } \\
\text { percibidos }\end{array}$ & $\begin{array}{l}\text { La MMA promueve la } \\
\text { mejora académica, la } \\
\text { competencia social, la } \\
\text { actividad física, la } \\
\text { regulación de las } \\
\text { emociones y la confianza } \\
\text { en sí mismos. }\end{array}$ & MMA & - \\
\hline $\begin{array}{l}\text { Luiselli et } \\
\text { al. (2017) }\end{array}$ & $\begin{array}{l}\text { Inglaterra. } \\
\text { Inglés }\end{array}$ & $\begin{array}{l}\text { Educación } \\
\text { Secundaria } \\
\text { (84 } \\
\text { estudiantes) }\end{array}$ & $\begin{array}{l}10 \\
\text { semanas } \\
(10 \\
\text { sesiones })\end{array}$ & $\begin{array}{l}\text { Cuantitativo } \\
: \\
\text { cuestionario } \\
\mathrm{s}\end{array}$ & $\begin{array}{l}\text { Evaluar la validez social } \\
\text { de la educación y las } \\
\text { prácticas de mindfulness } \\
\text { con estudiantes de } \\
\text { secundaria. }\end{array}$ & $\begin{array}{l}\text { Aceptación y satisfacción } \\
\text { con el programa }\end{array}$ & $\begin{array}{l}\text { El Mindfulness les ayudó a } \\
\text { ser más conscientes de sus } \\
\text { emociones, pensamientos y } \\
\text { sentimientos, así como a } \\
\text { estar más presentes en la } \\
\text { vida y a lidiar con el estrés } \\
\text { y los pensamientos } \\
\text { negativos. }\end{array}$ & - & - \\
\hline $\begin{array}{l}\text { O'Connor } \\
(2019)\end{array}$ & $\begin{array}{l}\text { Australia. } \\
\text { Inglés }\end{array}$ & $\begin{array}{l}\text { Educación } \\
\text { Secundaria } \\
\text { (44 } \\
\text { estudiantes) }\end{array}$ & $\begin{array}{l}12 \\
\text { semanas } \\
(12 \\
\text { sesiones })\end{array}$ & $\begin{array}{l}\text { Cualitativo: } \\
\text { entrevistas }\end{array}$ & $\begin{array}{l}\text { Por medio del } \\
\text { Mindfulness los } \\
\text { estudiantes debían } \\
\text { recordar en sus } \\
\text { experiencias anteriores } \\
\text { aquello que les motivara } \\
\text { de forma positiva a la } \\
\text { actividad física. }\end{array}$ & $\begin{array}{l}\text { Construcción de } \\
\text { signficado a través de } \\
\text { reflexión }\end{array}$ & $\begin{array}{l}\text { Los datos obtenidos } \\
\text { sugieren la reflexión y la } \\
\text { generación de narrativas } \\
\text { pueden mostrar aquello } \\
\text { que los estudiantes } \\
\text { encuentran significativo en } \\
\text { el movimiento, en formas } \\
\text { que vinculan la EF con } \\
\text { experiencias a través de } \\
\text { diferentes entornos sociales } \\
\text { y contextos. }\end{array}$ & - & - \\
\hline
\end{tabular}




\section{López Secanell, I.; Gené-Morales, J.}

\begin{tabular}{|c|c|c|c|c|c|c|c|c|c|}
\hline $\begin{array}{l}\text { Ullrich- } \\
\text { French et al. } \\
\text { (2017) } \\
\text { Estudio } 1\end{array}$ & $\begin{array}{l}\text { Estados } \\
\text { Unidos. }\end{array}$ & $\begin{array}{l}\text { Educación } \\
\text { Primaria y } \\
\text { Secundaria (15 } \\
\text { estudiantes) }\end{array}$ & $\begin{array}{l}3 \text { semanas } \\
\text { (1 sesión) }\end{array}$ & $\begin{array}{l}\text { Cualitativo: } \\
\text { entrevistas } \\
\text { cognitivas }\end{array}$ & $\begin{array}{l}\text { Evaluar cualitativa la } \\
\text { aplicación del cuestionario } \\
\text { MS-PA en la actividad } \\
\text { física en los estudiantes que } \\
\text { participaron en el estudio. }\end{array}$ & $\begin{array}{l}\text { Fiabilidad del } \\
\text { cuestionario } \\
\text { Necesidad de } \\
\text { reformular las preguntas } \\
\text { del cuestionario para } \\
\text { niños }\end{array}$ & $\begin{array}{l}\text { Los sujetos más jóvenes } \\
\text { tuvieron problemas para la } \\
\text { comprensión de algunas } \\
\text { preguntas del cuestionario } \\
\text { original }\end{array}$ & - & - \\
\hline $\begin{array}{l}\text { Ullrich- } \\
\text { French et al. } \\
\text { (2017) } \\
\text { Estudio } 2\end{array}$ & Inglés & $\begin{array}{l}\text { Educación } \\
\text { Primaria y } \\
\text { Secundaria (481 } \\
\text { estudiantes) }\end{array}$ & $\begin{array}{l}3 \text { semanas } \\
\text { (1 sesión) }\end{array}$ & $\begin{array}{l}\text { Cuantitativo: } \\
\text { Cuestionario }\end{array}$ & $\begin{array}{l}\text { Tras una sesión de yoga, } \\
\text { comparar dos versiones del } \\
\text { cuestionario MS-PA (A y } \\
\text { B) en niños y compararlas } \\
\text { con el MAAS. }\end{array}$ & $\begin{array}{l}\text { Validez del cuestionario } \\
\text { MS-PA }\end{array}$ & $\begin{array}{l}\text { La aplicación del } \\
\text { cuestionario MAAS mostró } \\
\text { una consistencia interna } \\
\text { marginal }(\alpha=0.64) \text { y el MS- } \\
\text { PA buena ( } \alpha=0.91-\mathrm{A} ; 0.92- \\
\text { B) }\end{array}$ & $\begin{array}{l}\text { Sesión de } \\
\text { actividad física } \\
\text { basada en yoga }\end{array}$ & - \\
\hline $\begin{array}{l}\text { Hagins y } \\
\text { Rundle } \\
\text { (2016) }\end{array}$ & $\begin{array}{l}\text { Estados } \\
\text { Unidos. } \\
\text { Inglés }\end{array}$ & $\begin{array}{l}\text { Educación } \\
\text { Secundaria y } \\
\text { Bachillerato (112 } \\
\text { estudiantes) }\end{array}$ & $\begin{array}{l}11 \text { meses } \\
\text { (48 } \\
\text { sesiones) }\end{array}$ & $\begin{array}{l}\text { Cuantitativo: } \\
\text { cuestionarios }\end{array}$ & $\begin{array}{l}\text { Examinar los efectos del } \\
\text { yoga escolar sobre el } \\
\text { rendimiento académico y } \\
\text { deportivo, así como, } \\
\text { explorar los posibles efectos } \\
\text { mediadores de la regulación } \\
\text { emocional. }\end{array}$ & $\begin{array}{l}\text { 1. Respuesta al estrés } \\
\text { 2. Función ejecutiva } \\
\text { auto-reportada } \\
\text { 3. Función ejecutiva } \\
\text { reportada por profesores } \\
\text { 4. Habilidades de } \\
\text { mindfulness } \\
\text { 5. Bienestar mental } \\
\text { 6. Cumplimiento de } \\
\text { actividades esenciales } \\
\text { en la escuela } \\
\text { 7. Media del curso }\end{array}$ & $\begin{array}{l}\text { El CAMM es una medida } \\
\text { apropiada para el desarrollo } \\
\text { de esta población con una } \\
\text { consistencia interna } \\
\text { adecuada. }\end{array}$ & $\begin{array}{l}\text { Programa de } \\
\text { yoga }\end{array}$ & - \\
\hline $\begin{array}{l}\text { Meixner et } \\
\text { al. (2019) }\end{array}$ & $\begin{array}{l}\text { Estados } \\
\text { Unidos. } \\
\text { Inglés }\end{array}$ & $\begin{array}{l}\text { Educación } \\
\text { Secundaria y } \\
\text { Bachillerato (24 } \\
\text { estudiantes) }\end{array}$ & $\begin{array}{l}20 \text { semanas } \\
(20 \\
\text { sesiones) }\end{array}$ & $\begin{array}{l}\text { Cualitativo: } \\
\text { entrevistas }\end{array}$ & $\begin{array}{l}\text { Explorar cualitativamente la } \\
\text { experiencia de los } \\
\text { estudiantes en MMA desde } \\
\text { la perspectiva de ellos y los } \\
\text { padres. }\end{array}$ & $\begin{array}{l}\text { 1. Fidelidad a las } \\
\text { características del } \\
\text { programa } \\
\text { 2. Presentación del } \\
\text { programa } \\
\text { 3. Comunicación }\end{array}$ & $\begin{array}{l}\text { La MMA promueve el } \\
\text { aumento de la participación } \\
\text { y el enfoque presente y } \\
\text { disminución de la falta de } \\
\text { interés de los desafíos. }\end{array}$ & MMA & \\
\hline
\end{tabular}

d: índice de Cohen índice de Cohen 